\title{
FORAMINÍFEROS BENTÓNICOS RECOLECTADOS DURANTE LA EXPEDICIÓN CIMAR 14 FIORDOS, PATAGONIA CHILENA
}

\author{
BENTHIC FORAMINIFERA COLLECTED DURING THE CIMAR \\ 14 FIORDS EXPEDITION, CHILEAN PATAGONIA
}

Tatiana Hromic M. ${ }^{1}$

\begin{abstract}
In 2008, the National Oceanographic Committee (CONA) of Chile, organised the CIMAR14 Fiords Expedition to the Chilean channels and fiords, located between $47^{\circ}$ and $50^{\circ} \mathrm{S}$. One of the groups studied was the benthic foraminifera. Information on the community structure of the group is reported and results are compared with those obtained in previous cruises.

The material studied comes from samples of superficial sea sediments, collected at 27 stations with a Box Corer. The samples were washed, dried and the microfauna extracted under a binocular microscope. Subsequently the species were identified and counted, assigning univariate and multivariate statistical indexes.

Overall, 2,569 shells of benthic foraminifera were obtained belonging to the following suborders: Rotaliina (60,8\%; 39 species); Textulariina (26,9\%; 19 species), Miliolina (6,4\%; 11 species), Lagenina $(5,77 \%)$, Involutinina $(1,97 \%)$, Spirillinina $(0,69 \%)$ and Robertinina $(0,42 \%)$ with one species each. Uvigerina bifurcata (13, $6 \%)$, Recurvoides scitullum (8,7 \%), Melonis affinis (6,8\%), Cassidulinoides parkerianus (5,2\%), Recurvoides contortus (5,2\%), Globobulimina notovata and Oridorsalis tener (4,3\%) were the most abundant. The species with a wider geographic distribution were G. notovata, Recurvoides contortus and $U$. bifurcata.

A conglomerate analysis allowed splitting the stations into four groups: 1) without defined geographic distribution, 2) coastal area, 3) southern area and 4) northern area.

Compared to the results obtained in previous surveys, there was an increase in the number of sand-foraminifera, and some species- mostly marine- were replaced by more euryhaline ones.

Key words: Benthic foraminifera; biodiversity; CIMAR; Channels; Fiords; Chile.

1 Laboratorio de Micropaleontología, Instituto de la Patagonia, Universidad de Magallanes. Casilla 113-D, Punta Arenas, Chile. tatiana.hromic@umag.cl
\end{abstract}




\section{RESUMEN}

Durante el año 2008, el Comité Oceanográfico Nacional (CONA), Chile, organizó la expedición CIMAR 14 Fiordos, a la zona de canales y fiordos chilenos, comprendida entre los $47^{\circ}$ y $50^{\circ} \mathrm{S}$. Uno de los grupos estudiados fue el de los foraminíferos bentónicos. En el presente trabajo se entregan antecedentes de la estructura comunitaria del grupo y se comparan los resultados con los obtenidos en cruceros previos.

El material estudiado proviene de muestras de sedimento marino superficial, obtenidas en 27 estaciones con un box corer. Las muestras fueron lavadas, secadas y la microfauna extraída bajo lupa binocular. Posteriormente se identificaron y contaron las especies, aplicándose índices estadísticos uni y multivariados.

En total se obtuvieron 2.569 caparazones de foraminíferos bentónicos, pertenecientes a los subórdenes: Rotaliina (60,8 \%; 39 especies); Textulariina (26,9\%; 19 especies), Miliolina (6,4 \%; 11 especies), Lagenina (5,77\%), Involutinina (1,97\%), Spirillinina (0,69\%) y Robertinina (0,42\%) con una especie cada uno. Las especies más abundantes fueron Uvigerina bifurcata (13,6 \%), Recurvoides scitullum (8,7 \%), Melonis affinis (6,8\%), Cassidulinoides parkerianus (5,2\%), Recurvoides contortus (5,2\%), Globobulimina notovata y Oridorsalis tener (4,3\%). Las especies con una distribución geográfica más amplia fueron $G$. notovata, Recurvoides contortus y U. bifurcata.

Un análisis de conglomerados permitió separar a las estaciones en 4 grupos: 1) sin situación geográfica definida, 2) área costera, 3) área sur y 4) área norte.

En relación con los resultados obtenidos en campañas previas hubo un incremento en el número de foraminíferos arenáceos, y especies típicamente marinas fueron reemplazadas por otras más eurihalinas.

Palabras clave: Foraminíferos bentónicos, biodiversidad, CIMAR, canales, fiordos, Chile.

\section{INTRODUCCIÓN}

La zona de fiordos y canales chilenos situada en el área subantártica, está prácticamente despoblada debido al difícil acceso y a las condiciones climáticas más bien desfavorables. La escasa influencia humana hacía de esta área una de las más prístinas del planeta. Sin embargo, el creciente interés económico asociado principalmente a la explotación de recursos marinos, en particular acuicultura, que se ha observado en las últimas décadas, hizo urgente definir líneas base de flora y fauna que en el futuro puedan medir el grado de impacto ambiental tras su explotación. Es así que el gobierno chileno, encargó al Comité Oceanográfico Nacional (CONA) organizar un conjunto de estudios, conocido como Programa CIMAR, bajo cuyo alero se llevan a cabo diversas expediciones científicas a la zona de canales y fiordos, con el objetivo de incrementar el conocimiento sobre su oceanografía, geomorfología, flora y fauna (Silva \& Palma 2006).

Uno de los grupos biológicos que ha podido ser estudiado más sistemática y profundamente, ha sido el de los foraminíferos bentónicos, llegando a identificarse unas 300 especies en el área comprendida entre Puerto Montt y Cabo de Hornos $\left(42^{\circ}-56^{\circ} \mathrm{S}\right)$ (Hromic 2001). En las últimas dos décadas aportes como los de Marchant (1993), Hromic (1999, 2001, 2005, 2009, 2010, 2011), Zapata \& Moyano (1993), Violanti et al. (2000), Zapata et al. (1995), entre otros, ha reunido una valiosa información sobre composición, abundancia, distribución, diversidad y ensambles faunísticos, generando una robusta base de datos. Kennet (1982) señaló que los foraminíferos han sido estudiados más que cualquier otro grupo de organismos marinos por su contribución a la reconstrucción de paleoambientes y que representan el 2,5\% de todas las especies animales conocidas desde el Cámbrico. El área de estudio desde el punto de vista oceanográfico es una zona compleja de tipo estuarino, que se caracteriza por una mezcla de aguas oceánicas de origen subantártico con aguas continentales derivadas del Campo de Hielo Patagónico Sur (Silva et al. 1998). El avance y retroceso de los hielos modeló el paisaje, dejando profundos surcos en forma de fiordos con aguas apozadas, a veces con difícil intercambio con aguas marinas, que dificultan el establecimiento de los foraminíferos. 
El objetivo de este trabajo es dar a conocer los resultados obtenidos del análisis de las muestras recolectadas durante CIMAR 14 Fiordos (2008) y compararlos con los resultados obtenidos en 1996, durante la expedición CIMAR 2 Fiordos.

\section{MATERIAL Y MÉTODOS}

Se recolectó una muestra de sedimento superficial en cada una de las 27 estaciones visitadas durante la expedición CIMAR 14 Fiordos, a los canales patagónicos chilenos $\left(47^{\circ}-50^{\circ} \mathrm{S}\right)$ y organizada por el Comité Oceanográfico Nacional (CONA) (Tabla 1; Fig.1).

Las muestras fueron lavadas sobre un tamiz de malla 63 micras, secadas y pesadas siguiendo la metodología de Boltovskoy (1965). Los especímenes fueron separados uno a uno bajo lupa binocular, pegados en reglillas por especie e identificados hasta el nivel genérico siguiendo a Loeblich \& Tappan
(1988) y hasta el nivel específico, la literatura del área (Hromic 2001, 2002, 2004, 2005; Hromic et al. 2006, Violanti et al. 2000, Marchant 1993, Zapata et al. 1995, etc.) y el catálogo de Ellis \& Messina (1940 et seq.). El recuento de ejemplares dio origen a una matriz de valores absolutos o matriz de datos (MA), la que fue transformada a valores de ejemplares por gramo (MEG) debido a la heterogeneidad de pesos de las muestras. La MEG fue transformada a raíz cuarta para minimizar las diferencias entre especies más y menos abundantes (Clarke \& Warwick 1994).

Para evidenciar diferencias y semejanzas entre las comunidades de foraminíferos bentónicos de las distintas estaciones se utilizaron tanto técnicas univariadas como multivariadas. Dentro de los análisis univariados, se determinó: a) composición taxonómica, b) densidad (ejemplares/gramo= N), c) riqueza de especies ( $\mathrm{n}^{\circ}$ de especies $=\mathrm{S}$ ), d) frecuencia $(\mathrm{n}$ - de estaciones en la que registraron cada una de las especies), e) diversidad ( $\mathrm{H}^{\prime}=$ =índice de Shannon-

Tabla 1. Lista de estaciones, área geográfica, ubicación y peso (g) de las muestras.

\begin{tabular}{|c|c|c|c|c|}
\hline Estación & Área geográfica & Latitud S & Longitud W & Peso \\
\hline C14 st. 6 & Canal Baker & $48^{\circ} 01,00^{\prime}$ & $74^{\circ} 19,30^{\prime}$ & 0,624 \\
\hline C14 st. 7 & Canal Baker & $47^{\circ} 59,30^{\prime}$ & $74^{\circ} 04,30^{\prime}$ & 0,402 \\
\hline C14 st. 9 & Canal Baker & $48^{\circ} 04,50^{\prime}$ & $73^{\circ} 38,20^{\prime}$ & 0,205 \\
\hline C14 st. 11 & Canal Troya & $47^{\circ} 55,05^{\prime}$ & $73^{\circ} 47,80^{\prime}$ & 0,313 \\
\hline C14 st. 12 & Caleta Tortel & $47^{\circ} 51,40^{\prime}$ & $73^{\circ} 35,90^{\prime}$ & 1,832 \\
\hline C14 st. 13 & Isla Teresa & $47^{\circ} 49,90^{\prime}$ & $73^{\circ} 40,95^{\prime}$ & 0,229 \\
\hline C14 st. 15 & Estero Steffen & $47^{\circ} 41,50^{\prime}$ & $73^{\circ} 43,30^{\prime}$ & 0,340 \\
\hline C14 st. 16 & Estero Mitchell & $47^{\circ} 59,38^{\prime}$ & $73^{\circ} 22,55^{\prime}$ & 0,207 \\
\hline C14 st. 18 & Canal Messier & $48^{\circ} 30,00^{\prime}$ & $74^{\circ} 30,00^{\prime}$ & 3,031 \\
\hline C14 st. 19 & Canal Messier & $48^{\circ} 39,00^{\prime}$ & $74^{\circ} 22,60^{\prime}$ & 0,221 \\
\hline C14 st. 20 & Angostura Inglesa & $48^{\circ} 53,10^{\prime}$ & $74^{\circ} 25,70^{\prime}$ & 0,401 \\
\hline C14 st. 22 & Paso del Indio & $49^{\circ} 02,80^{\prime}$ & $74^{\circ} 25,80^{\prime}$ & 1,838 \\
\hline C14 st. 23 & Paso del Indio & $49^{\circ} 11,20^{\prime}$ & $74^{\circ} 22,50^{\prime}$ & 1,042 \\
\hline C14 st. 25 & Canal Icy & $49^{\circ} 33,50^{\prime}$ & $74^{\circ} 12,60^{\prime}$ & 0,221 \\
\hline C14 st. 26 & Seno Eyre & $49^{\circ} 31,60^{\prime}$ & $74^{\circ} 04,30^{\prime}$ & 0,809 \\
\hline C14 st. 27 & Seno Eyre & $49^{\circ} 23,10^{\prime}$ & $74^{\circ} 04,90^{\prime}$ & 1,851 \\
\hline C14 st. 29 & Estero Falcon & $49^{\circ} 32,00^{\prime}$ & $73^{\circ} 58,00^{\prime}$ & 3,805 \\
\hline C14 st. 31 & Canal Wide & $49^{\circ} 47,80^{\prime}$ & $74^{\circ} 21,90^{\prime}$ & 0,506 \\
\hline C14 st. 35 & Canal Wide & $49^{\circ} 58,10^{\prime}$ & $74^{\circ} 25,80^{\prime}$ & 2,083 \\
\hline C14 st. 85 & Canal Picton & $49^{\circ} 28,90^{\prime}$ & $75^{\circ} 25,15^{\prime}$ & 3,350 \\
\hline C14 st. 87 & Canal Ladrillero & $49^{\circ} 06,24^{\prime}$ & $75^{\circ} 15,25^{\prime}$ & 2,338 \\
\hline C14 st. 88 & Canal Ladrillero & $48^{\circ} 56,50^{\prime}$ & $75^{\circ} 02,00^{\prime}$ & 0,603 \\
\hline C14 st. 89 & Canal Fallos & $48^{\circ} 39,20^{\prime}$ & $74^{\circ} 59,20^{\prime}$ & 4,311 \\
\hline C14 st. 90 & Canal Fallos & $48^{\circ} 23,40^{\prime}$ & $75^{\circ} 06,50^{\prime}$ & 20,00 \\
\hline C14 st. 91 & Canal Fallos & $48^{\circ} 04,15^{\prime}$ & $75^{\circ} 17,00^{\prime}$ & 0,502 \\
\hline C14 st. 93 & Golfo de Penas & $47^{\circ} 22,20^{\prime}$ & $74^{\circ} 38,70^{\prime}$ & 1,094 \\
\hline C14 st. 95 & Bahía San Quintín & $46^{\circ} 48,85^{\prime}$ & $74^{\circ} 26,95^{\prime}$ & 20,00 \\
\hline
\end{tabular}




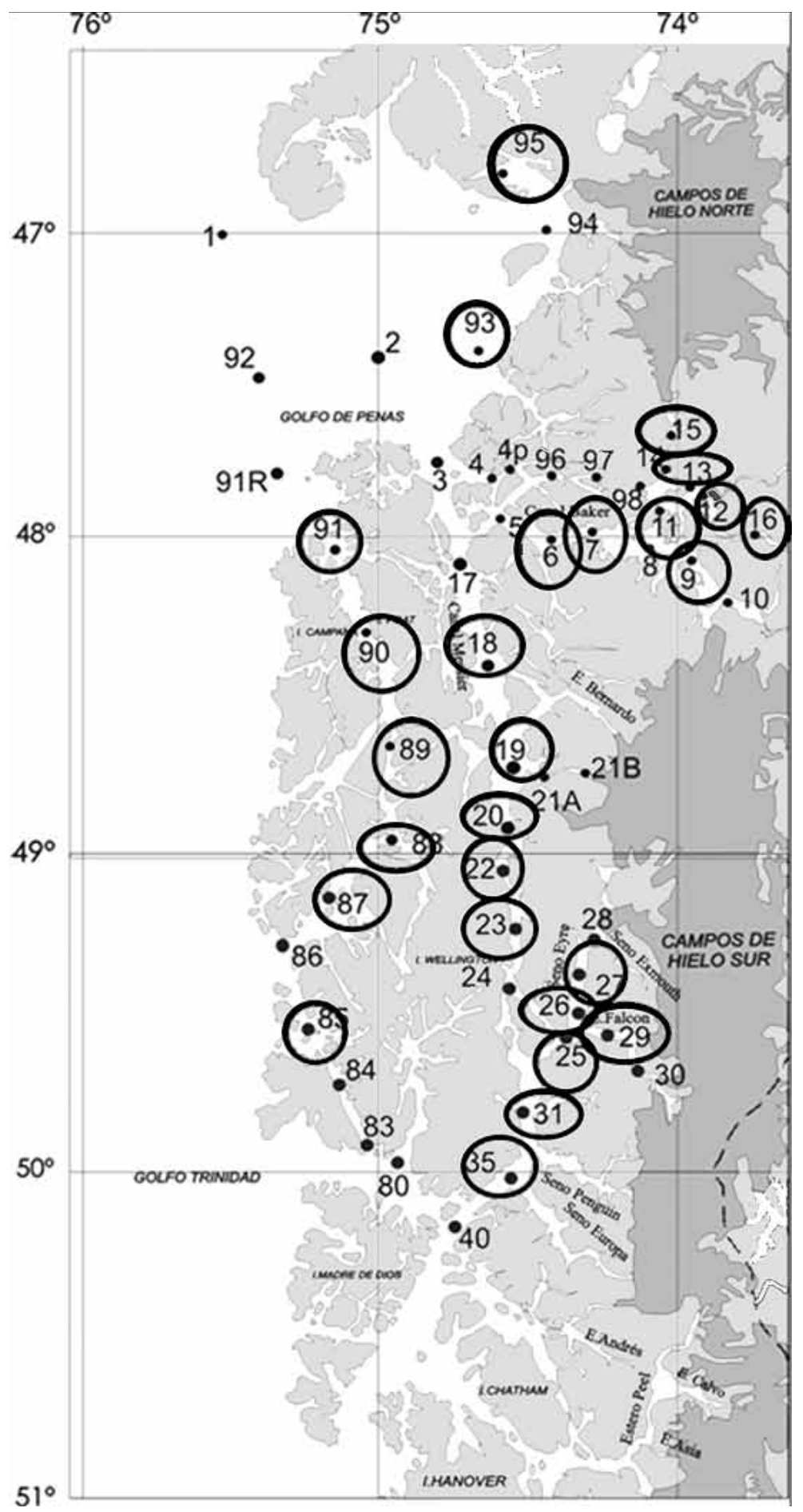

Fig. 1. Localización de las muestras estaciones de muestreo (en círculo). 
Wiener) y f) equidad (J' =índice de Pielou, Magurran 1988). Respecto de este último, cabe destacar que mide la proporción de la diversidad observada en relación con la diversidad máxima esperada. Sus valores varían entre 0 y 1 ; este último valor corresponde a situaciones donde todas las especies son igualmente abundantes (Moreno et al. 2001).

Las técnicas multivariadas incluyeron la clasificación jerárquica utilizando para ello el índice de Bray-Curtis (1957). Se construyó una matriz de similitud (MS) de especies, aplicando SIMPROF. A partir de ésta, se graficaron los resultados en un dendrograma. Los grupos formados fueron evaluados mediante un gráfico de ordenamiento de muestras usando la técnica MDS (Escalamiento Multidimensional), (Clarke 1993, Clarke \& Green 1988, Ludwig \& Reynold 1988). La bondad de ajuste se verificó teniendo en consideración el stress (Kruskal \& Carmone 1971) que mide la calidad de la representación. Valores $<0,1$ señalan una muy buena ordenación y valores $>0,2$ una ordenación menos confiable (Clarke \& Warwick 1994). La consistencia entre los resultados de ambas técnicas sugiere la robustez del análisis (Wood-Walker et al. 2002). A continuación se aplicó el análisis de similitud porcentual SIMPER (Clarke \& Warwick 1994) sobre la MEG, para calcular los porcentajes de disimilitud entre los grupos y similitud al interior de los grupos e identificar las especies que más contribuyeron a los resultados.

Considerando que estos análisis son solamente exploratorios, la confirmación estadística se obtuvo aplicando la prueba Análisis de Similitud (ANOSIM) de una vía a un nivel de significancia del 5\%, la que permitió verificar si las agrupaciones resultantes de los análisis de conglomerados y MDS presentaban diferencias significativas entre sí. Cuando los valores de $\mathrm{R}$ resultantes son iguales o cercanos a 1 , sugiere que los grupos comparados presentan diferencias, por el contrario si se acercan a -1 los grupos son similares entre sí. Todas estas pruebas se hicieron con el paquete PRIMER 6.0 desarrollado por el Plymouth Marine Laboratory, U.K. (Clarke \& Gorley 2006).

\section{RESULTADOS}

\section{Composición taxonómica}

Se recolectó un total de 2.569 foraminíferos bentónicos, los que fueron clasificados en siete subórdenes: Rotaliina, Textulariina, Miliolina, Lagenina, Involutinina, Spirillinina y Robertinina. Los subórdenes Rotaliina (60,8\%), Textulariina (26, $9 \%)$ y Miliolina $(6,4 \%)$ contribuyeron con el mayor número de ejemplares (Fig. 2).

Dentro del suborden Rotaliina se identificaron 39 especies (44,3\%), siendo Uvigerina bifurcata (22,3\%), Melonis affinis (11,1\%), Cassidulinoides parkerianus (8,5\%), Globobulimina notovata (7,1 \%) y Oridorsalis tener (7\%), las especies con mayor representación. El suborden Textulariina aportó 19 especies $(21,6 \%)$ y las especies dominantes fueron Recurvoides scitulum (32,2\%), Recurvoides contortus (16,6\%), Cyclammina cancellata (9,3\%), Rhizammina

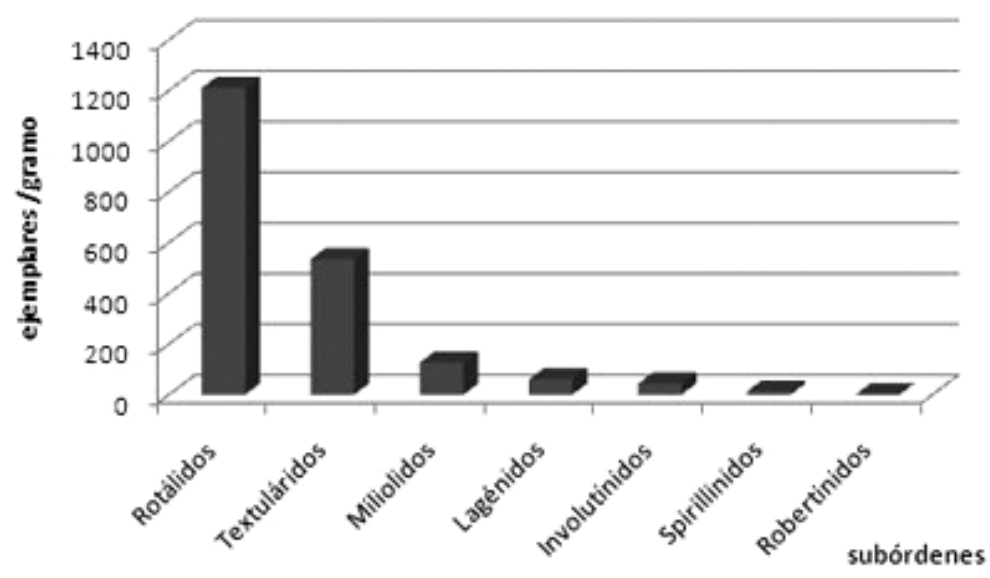

Fig. 2. Número de ejemplares/gramo recolectados de cada uno de los subórdenes. 


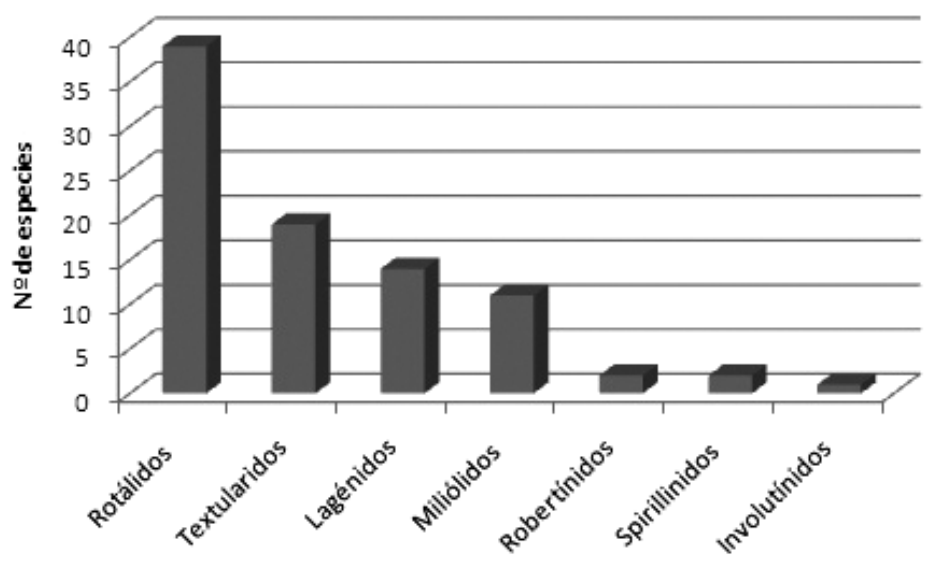

Fig. 3. Número de especies identificadas en cada uno de los subórdenes.

algaeformis (8,4 \%) y Labrospira kosterensis (8\%). Dentro de Lagenina que contribuyó con el 15,9\% de las especies (14 formas), destacaron Dentalina consobrina (48,7\%), Lenticulina orbicularis $(12,7$ \%) y Lagena clavata (9,3 \%). El suborden Miliolina aportó el 12,5\% de las especies (11 especies) y se identificaron como especies dominantes, Spiroloculina tenuis (39\%), Quinqueloculina seminula (20,5\%) y Miliolinella subrotunda (11,3\%). Robertínidos y Spirillínidos aportaron con el 2,3\% de las especies cada uno. Los robertínidos estuvieron representados por Robertina sp. (78,3 \%) y Hoeglundina elegans $(21,7 \%)$ y Spirillínidos, por Spirillina vivipara respectivamente. Finalmente, el suborden Involutina $(1,84 \%)$ tuvo un único representante: Involutina tenuis (Fig. 3).

El 81,8\% de las especies estuvo pobremente representada $(<2 \%)$ en el área. La lista de las 16 especies más abundantes (> $2 \%$ ) en el área y su frecuencia se presentan en la tabla 2 . De estas 16, más de la mitad de las especies (56,3\%) se clasificaron dentro del suborden Rotaliina. Los rotálidos

Tabla 2. Abundancia relativa (\%) y frecuencia de las especies dominantes (> $2 \%$ ).

\begin{tabular}{|c|c|c|}
\hline & Abundancia relativa & Frecuencia $\mathrm{n}^{\circ}$ estaciones \\
\hline Uvigerina bifurcata d'Orbigny, 1839 & 13,57 & 11 \\
\hline $\begin{array}{l}\text { Recurvoides scitullum (Brady) } \\
\quad=\text { Haplophragmoides scitullum Brady, } 1881\end{array}$ & 8,67 & 9 \\
\hline Melonis affinis (Reuss, 1851) & 6,75 & 10 \\
\hline $\begin{array}{l}\text { Cassidulinoides parkerianus (Brady) } \\
=\text { Cassidulina parkeriana Brady, } 1884 \text {. }\end{array}$ & 5,19 & 4 \\
\hline Recurvoides contortus Earland, 1934 & 4,47 & 11 \\
\hline $\begin{array}{l}\text { Globobulimina notovata (Chapman) } \\
\text { = Bulimina notovata Chapman, } 1941\end{array}$ & 4,30 & 14 \\
\hline Oridorsalis tener (Brady, 1884) & 4,27 & 9 \\
\hline $\begin{array}{l}\text { Globocassidulina minuta (Cushman) } \\
=\text { Cassidulina minuta Cushman, } 1933\end{array}$ & 4,17 & 8 \\
\hline Cassidulina laevigata d'Orbigny, 1826. & 3,17 & 5 \\
\hline $\begin{array}{l}\text { Angulogerina angulosa (Williamson) } \\
=\text { Uvigerina angulosa Williamson, } 1858 .\end{array}$ & 2,60 & 9 \\
\hline Cyclammina cancellata Brady, 1879 & 2,49 & 10 \\
\hline $\begin{array}{l}\text { Spiroloculina tenuis (Czjzek) } \\
\quad=\text { Quinqueloculina tenuis Czjzek, } 1848\end{array}$ & 2,48 & 9 \\
\hline Nonionella sp a & 2,33 & 7 \\
\hline Rhizammina algaeformis Brady, 1879 & 2,26 & 6 \\
\hline $\begin{array}{l}\text { Involutina tenuis (Brady) } \\
\quad=\text { Ammodiscus tenuis Brady } 1884\end{array}$ & 2,16 & 10 \\
\hline Labrospira kosterensis Höglund, 1947 & 2,14 & 5 \\
\hline
\end{tabular}




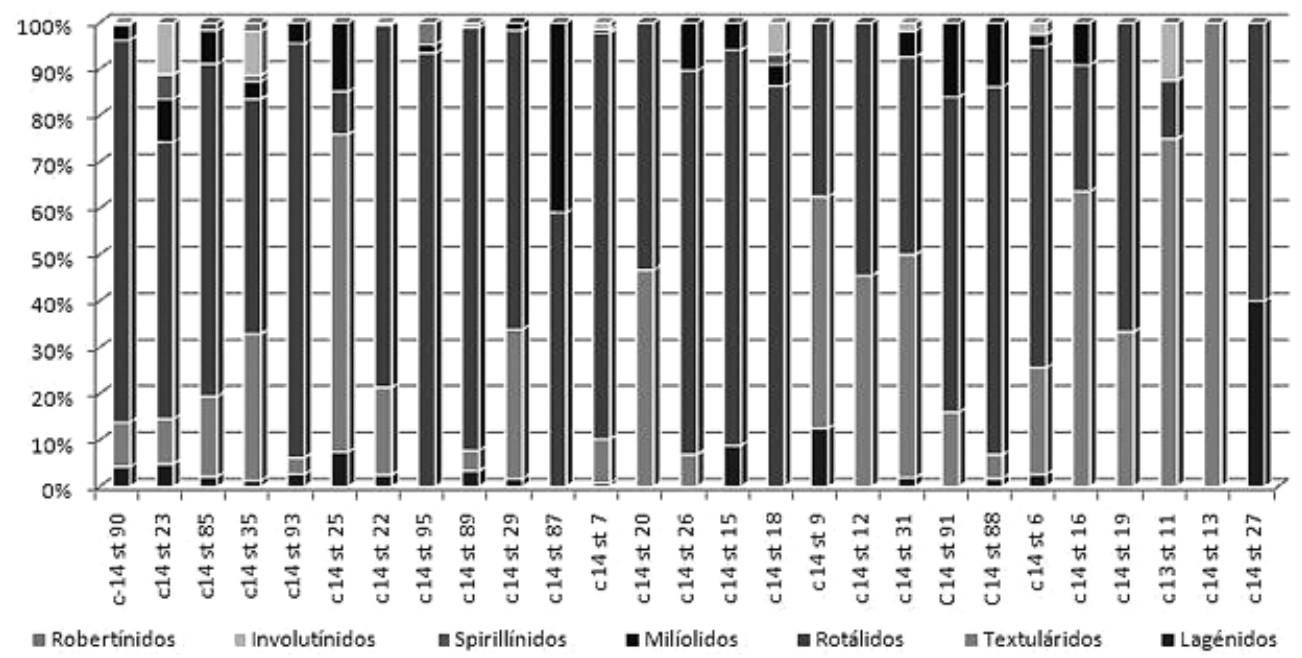

Fig. 4. Relación porcentual de los subórdenes en las estaciones muestreadas.

Uvigerina bifurcata y Globobulimina notovata fueron las especies más abundantes en el área y las que se distribuyeron en el mayor número de estaciones. El $31 \%$ de las especies de la lista, se clasificaron en el suborden Textulariina, y el 6,3\%, en miliólidos e involutínidos respectivamente. Cabe destacar que ningún lagénido, spirillínido ni robertínido alcanzó una representación superior al 2\%. La única especie de Lagenina que se presentó en un alto número de estaciones (9) fue Dentalina consobrina, sin embargo el número de ejemplares fue de 1,5\%.

En relación con la distribución de subórdenes por estación se observa claramente el dominio de rotálidos y textuláridos en todas ellas (Fig. 4). Los textuláridos se presentaron mayoritariamente en las estaciones № 25 (canal Icy), № 9 (canal Baker), № 16 (estero Mitchell), № 11 (canal Troya) y № 13 (isla Teresa), todas situadas en el interior de los canales (Fig. 4). Los miliólidos estuvieron presentes en la mayoría de las estaciones con bajo número de ejemplares y ausentes en las estaciones № 9 , № 11 , № 12 , № 13 , № 19 , № 20 , № 22 y № 27 .

El número de ejemplares por gramo en cada muestra fue muy heterogéneo. En promedio se contabilizaron 73,73 ejemplares/gramo, siendo los valores máximo y mínimo, 340,79 ejemplares/gramo (estación № 7, canal Baker) y 2,71 ejemplares / gramo (estación № 27, seno Eyre) respectivamente.

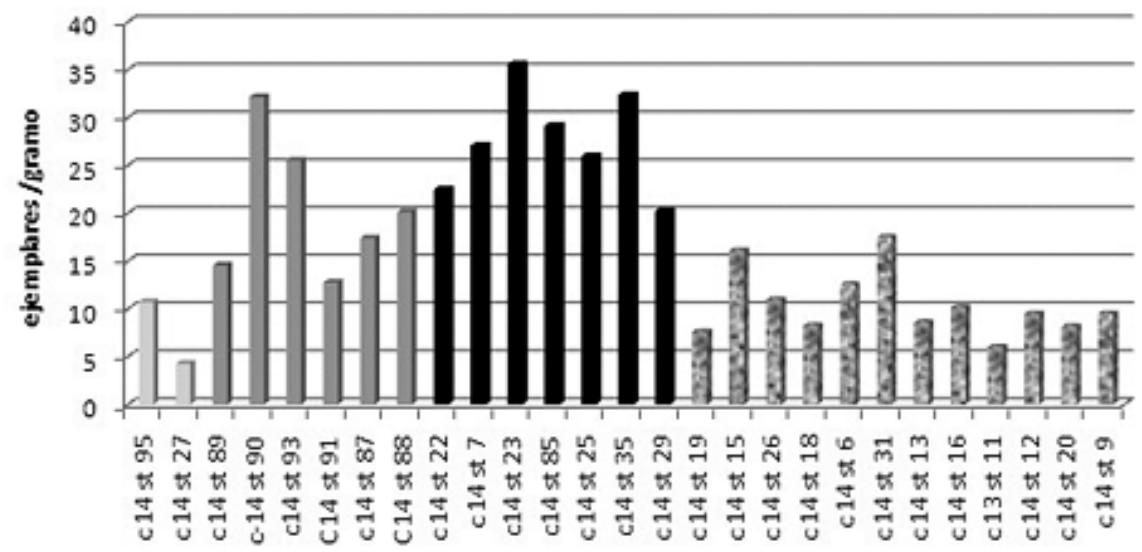

Fig. 5. Densidad expresada como número de ejemplares por gramo. Las distintas tonalidades de gris representan los grupos obtenidos según análisis de cluster. 


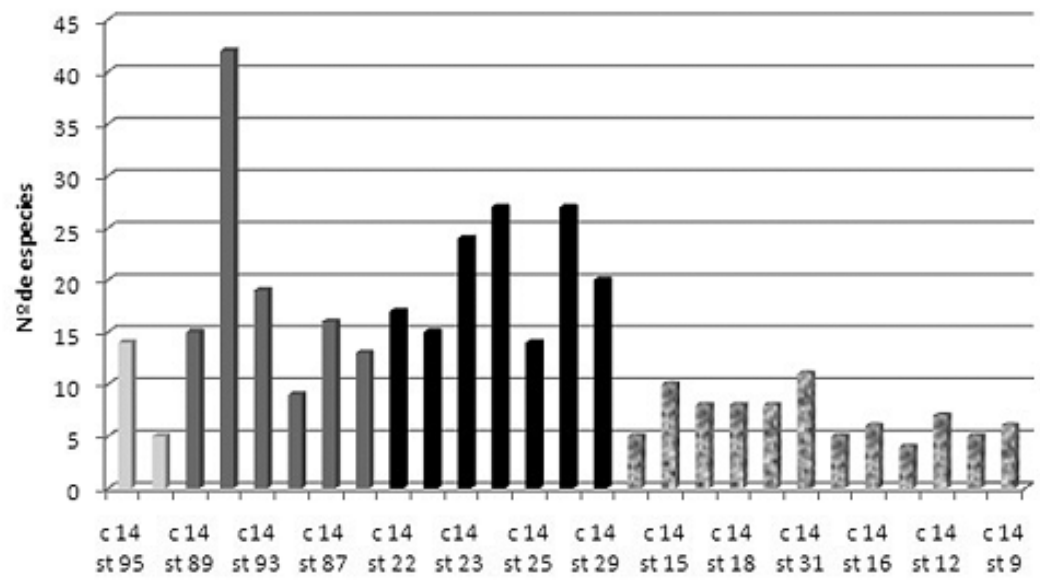

Fig. 6. Riqueza de especies.

En las estaciones № 90 (canal Fallos), № 23 (Paso del Indio) y № 35 (canal Wide) se registró la mayor cantidad de ejemplares, mientras que en las estaciones № 27 (canal Eyre), № 19 (canal Messier) y № 11 (canal Troya) la menor cantidad (Fig. 5). De acuerdo al agrupamiento obtenido en el análisis de cluster (ver Fig. 10), las muestras del área sur son las que contienen el mayor número de ejemplares/ gramo.

\section{Riqueza de especies}

Se identificó un total de 88 especies de foraminíferos bentónicos en el área de estudio. El número de especies en las distintas muestras varió considerablemente desde 42 especies en la muestra recolectada en la estación № 90 hasta 3 especies en la estación № 11 (Fig. 6). El número de especies promedio más alto se registró en el área sur.

\section{Frecuencia}

La distribución geográfica de las especies fue más bien restringida, esto es, el 39,8 \% de la especies solo se encontraron presentes en una 0 dos estaciones y el $35 \%$ en dos a 5 estaciones (Fig. 7). Ello significa que el $75 \%$ de las especies solo se encuentra en el 18, $5 \%$ de las estaciones, mientras que el $25 \%$ alcanza una distribución más alta que no sobrepasa la mitad de las estaciones.

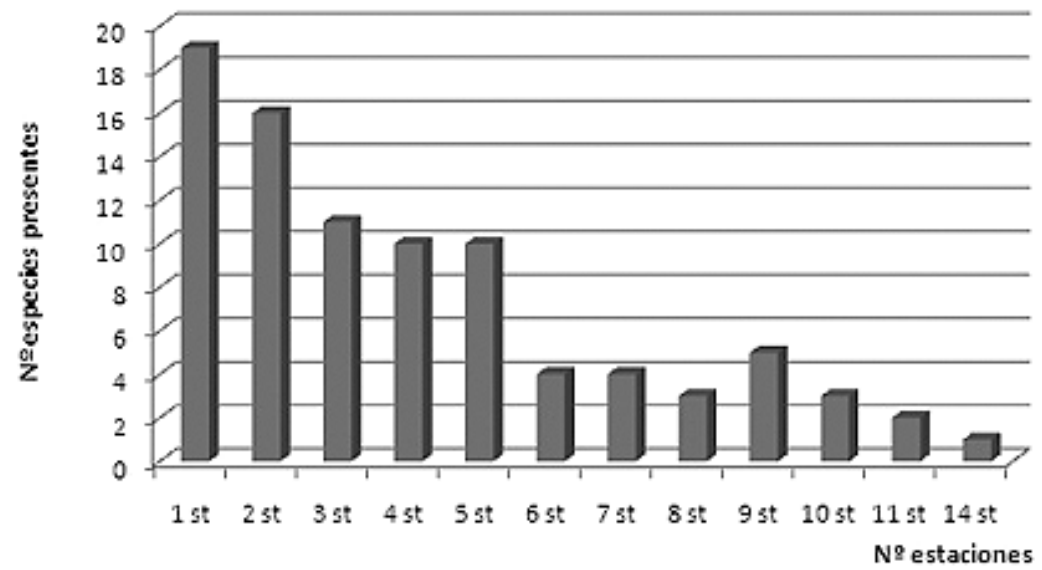

Fig. 7. Frecuencia expresada como número de especies versus número de estaciones. 


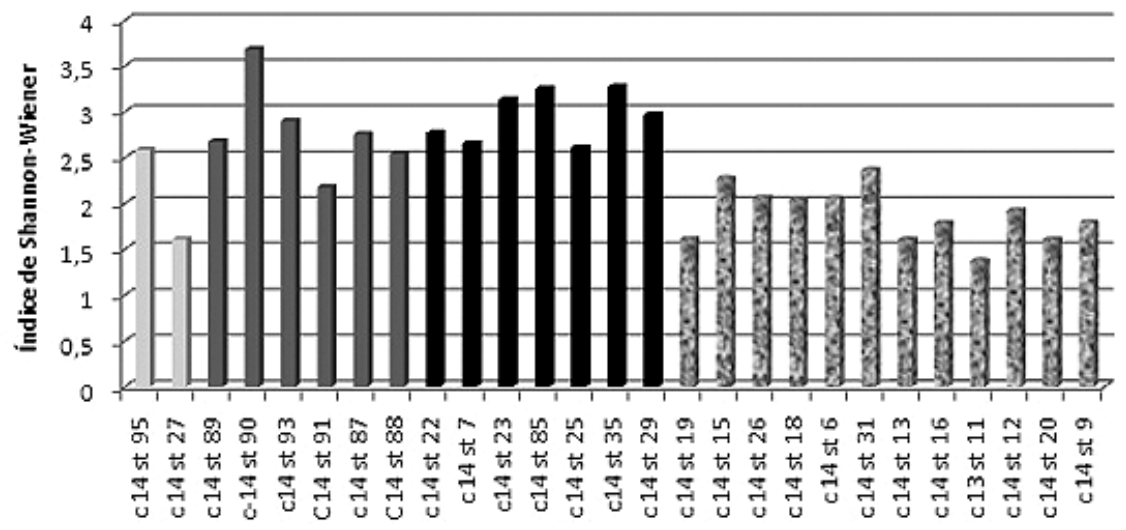

Fig. 8. Diversidad (índice de Shannon-Wiener, H').

Solo una especie, Globobulimina notovata, se presentó en la mitad de las estaciones (14 estaciones), y dos: Recurvoides contortus y Uvigerina bifurcata en 11 estaciones, el resto de especies solo se registró en menos de la mitad de las estaciones.

\section{Diversidad}

El índice de diversidad fue mayor de 2 en el 59,3\% de las estaciones. La diversidad más alta se alcanzó en la estación № 90 y la más baja en la estación № 11 (Fig. 8). La diversidad promedio más alta se encontró en las estaciones del área sur.

\section{Equidad}

El índice de Pielou (> 0,965) evidencia que los ensambles son estables y que no hay dominancia clara de algunas especies (Fig. 9).

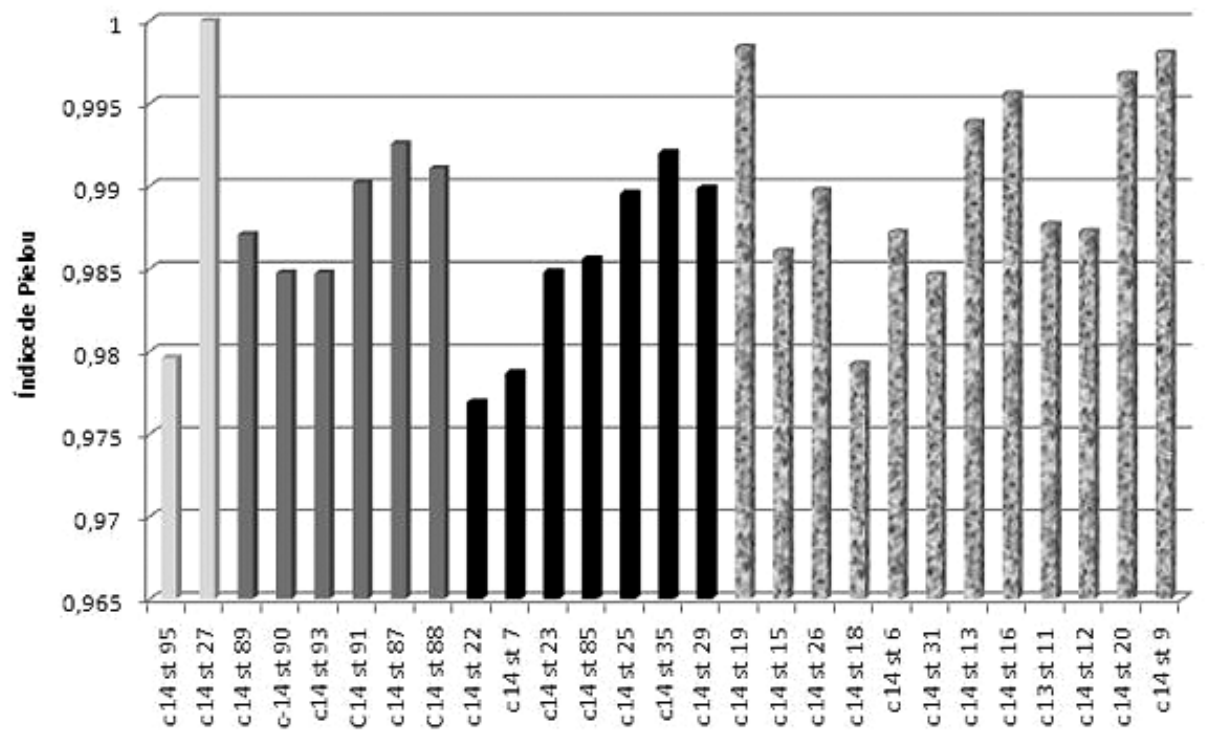

Fig. 9. Equidad (índice de Pieolu, J'). 


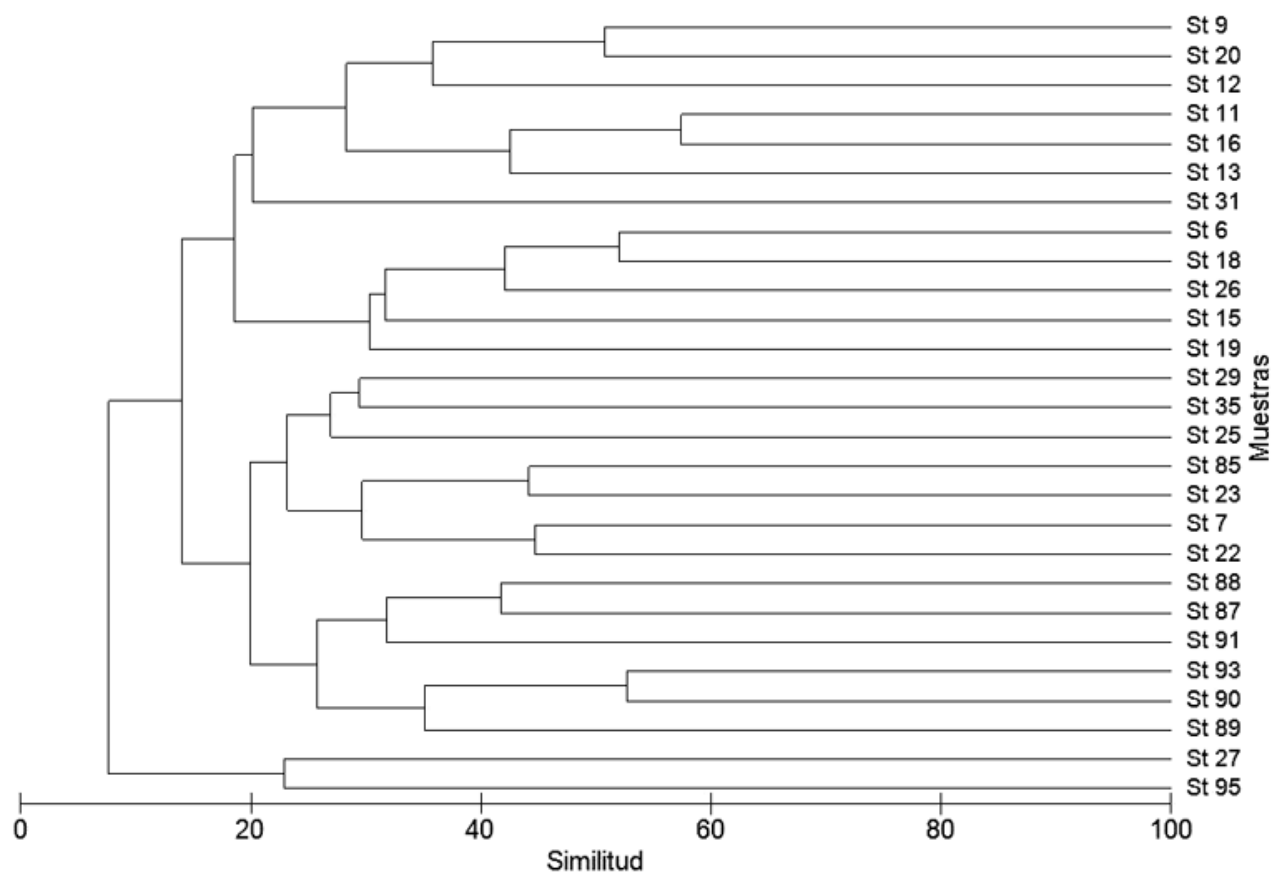

Fig. 10. Análisis de conglomerados (Índice de Bray- Curtis).

Análisis de conglomerados

El análisis de conglomerados se realizó para identificar la asociación entre las estaciones. Con ca. $20 \%$ de similitud las agrupaciones pueden considerarse débiles, es decir, las muestras no son tan parecidas en término de abundancia y especies. No obstante, se distinguieron 4 grupos de estaciones. Este agrupamiento fue confirmado por el análisis
MDS con un valor de stress de 0,23, que si bien es algo alto puede considerarse dentro del rango aceptable (Figs. 10 y 11).

El primer grupo (1) reunió las muestras obtenidas en las estaciones № 95 (bahía San Quintín) y № 27 (seno Eyre), lejanas geográficamente pero situadas en zonas internas de los canales.

Un segundo grupo (2) estuvo conformado por 6 muestras obtenidas en las estaciones № 89 , №

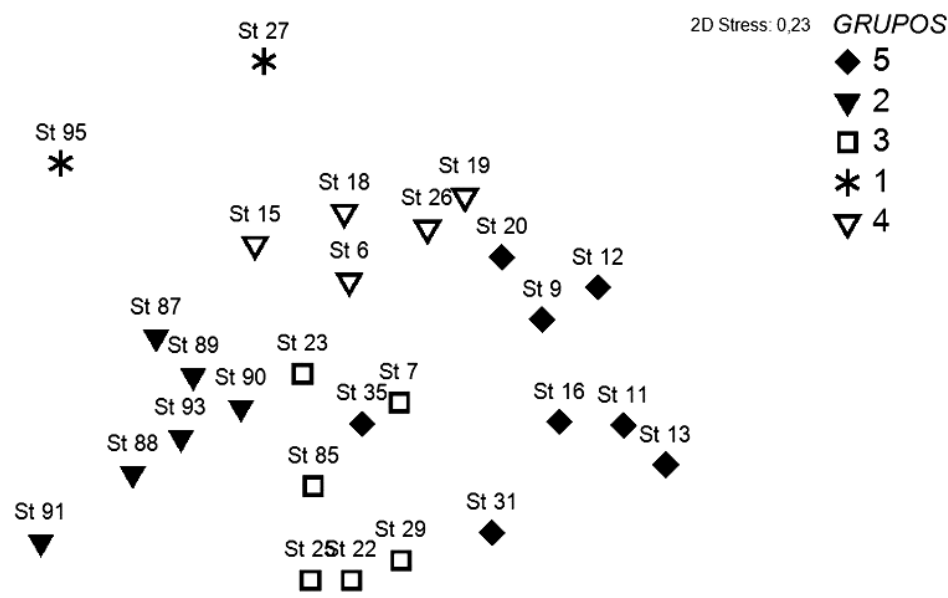

Fig. 11. Ordenamiento de las muestras obtenidas durante la expedición CIMAR 14 fiordos, con la técnica MDS. 
90 y № 91 (canal Fallos), № 93 (golfo de Penas), № 87 y № 88 (canal Ladrilleros), situadas en las cercanías del océano.

Un tercer grupo (3) reunió las muestras tomadas en las estaciones № 22 y № 23 (Paso del Indio), № 7 (canal Baker), № 85 (canal Picton), № 25 (canal Icy), № 35 (canal Wide) y № 29 (Estero Falcón), localizadas en la sección más meridional excepto la muestra obtenida en la estación № 7 situada sobre el canal Baker, de los canales intermedios.

Un cuarto y último grupo (4) que integró el mayor número de muestras estuvo conformado por el material recolectado en las estaciones № 18 y № 19 (canal Messier), № 15 (Estero Steffen), № 26 (Seno Eyre), № 6 y № 9 (canal Baker), № 31 (canal Wide), № 13 (isla Teresa), № 16 (estero Mitchell), № 11 (canal Troya), № 12 (caleta Tortel) y № 20 (Angostura Inglesa), situadas sobre el canal Baker y Messier, en la porción norte.

Como resultado del análisis de similitud ANOSIM se determinó que hubo diferencias significativas entre los grupos, con un $\mathrm{R}$ global de 0,57 a un nivel de significancia $0,1 \%$.

\section{Especies indicadoras}

Como resultado del análisis de similitud porcentual SIMPER se definieron las especies tipificadoras de cada grupo y discriminadoras entre grupos.

Las especies responsables del agrupamiento de las estaciones se indican a continuación.

\section{i) 1.Similitud}

Para el grupo 1, conformada por las estaciones № 95 y № 27, muy apartadas entre sí, el promedio de similitud fue de $22,9 \%$ y si bien considera solo dos muestras, las especies tipificadoras fueron Cibicidinella variabilis y Nonionella auris.

Para el grupo 2 ("zona borde oceánico"), con un promedio de similitud de 30,63\%, las especies responsables fueron Oridorsalis tener que contribuye con el 21,8\%, Spiroloculina tenuis $(12,96$ $\%)$, Angulogerina angulosa (7,1\%) y Discorbinella bertheloti (6,4\%).

En el grupo 3 ("zona sur") con un promedio de similitud de 27,1 \% las especies que más aportaron al agrupamiento fueron: Angulogerina angulosa (9,6\%), Uvigerina bifurcata (8,3\%), Reophax scorpiurus (6,4\%), Dentalina consobrina $(6,1 \%)$ y Globocassidulina minuta (6,1\%). Dentro de este grupo cabe destacar que del total de especies que contribuyen al 70\% de la similitud, diez tenían caparazón calcáreo y 5 caparazón arenáceo.

Finalmente para el cuarto grupo ("zona norte"), el promedio de similitud fue de $24,9 \%$ y las especies responsables de la similitud fueron Globobulimina notovata (25,6\%), Recurvoides contortus (20\%), Cyclammina cancellata (10,2\%). Nonionella sp. a y Melonis affinis $(9,9 \%)$ y $(7,7 \%)$ respectivamente.

\section{i.2. Disimilitud}

La disimilitud entre los grupos dos y tres fue dada por la contribución de 23 especies discriminatorias (70 \% caparazón calcáreo y 30 \% caparazón arenáceo) que contribuyeron con el 50 \% y cuyas contribuciones particulares fluctuaron entre 1,67 \% y $3,7 \%$.

La disimilitud entre los grupos uno y dos estuvo dada por 16 especies (solo una con caparazón arenáceo), las que contribuyeron con el 51,4 \%. La contribución porcentual de cada una de las especies osciló entre 2,3\% y 6,3\%.

La disimilitud entre los grupos uno y tres estuvo dada por 20 especies (75 \% con caparazón calcáreo y $25 \%$ con caparazón arenáceo) que contribuyeron con el 51,3\%.y cuyas contribuciones parciales fueron del 1,84 \% al 4,6\%.

La disimilitud entre los grupos dos y cuatro estuvo dada por 16 especies (81,3\% con caparazón arenáceo y 18,7 \% con caparazón calcáreo), las cuales contribuyeron con el 51,9 \%. Las especies contribuyeron de manera particular con porcentajes entre $2,4 \%$ y $5,6 \%$.

La disimilitud entre los grupos tres y cuatro estuvo dada por 18 especies (11 con caparazón calcáreo (61 \%) y 7 con caparazón arenáceo (38\%)) que contribuyeron con el 51,9 \% de la disimilitud. La especie discriminatoria con mayor participación fue Uvigerina bifurcata con $4,7 \%$ y la que menos contribuyó fue Nonionella auris, con 2,02\%.

Finalmente, la disimilitud entre los grupos uno y cuatro estuvo dada por 11 especies (8 con caparazón calcáreo y 3 con caparazón arenáceo) que contribuyeron al $51 \%$ de la disimilitud. La especie que contribuyó más fue Recurvoides contortus (6,3\%) y la que menos contribuyó Nonionella chilensis (3,3\%).

Los resultados del análisis de disimilitud se muestran en la tabla 3. 
Tabla 3. Valores de disimilitud y principales especies discriminadoras entre grupos (SIMPER, PRIMER).

\begin{tabular}{|c|c|c|c|}
\hline & Grupo 1 & Grupo 2 & Grupo 3 \\
\hline \multirow{4}{*}{ 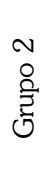 } & 92,84 & & \\
\hline & Oridorsalis tener $6,31 \%$ & & \\
\hline & Spiroloculina tenuis $4,76 \%$ & -- & --- \\
\hline & Nonionella auris 3,87\% & & \\
\hline \multirow{4}{*}{ 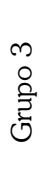 } & 92,30 & 80,09 & \\
\hline & Uvigerina bifurcata $(4,62 \%)$ & U. bifurcata, $3,7 \%$ & \\
\hline & Angulogerina angulosa $(3,68 \%)$ & Oridorsalis tener (3,36\%) & --- \\
\hline & Globocassidulina minuta $(3,27 \%)$ & Recurvoides scitulum (2,78\%) & \\
\hline \multirow{4}{*}{$\begin{array}{l}\text { मे } \\
\text { ڤ్ } \\
\text { 今్ }\end{array}$} & 92,24 & 89,18 & 83,44 \\
\hline & Recurvoides contortus $(6,31 \%)$ & Oridorsalis tener $(5,55 \%)$ & Uvigerina bifurcata $(4,58 \%)$ \\
\hline & Globobulimina notovata $(5,9 \%)$ & Spiroloculina tenuis $(4,21 \%)$ & Angulogerina angulosa $(3,74 \%)$ \\
\hline & Cibicidinella variabilis $(5,8 \%)$ & Globobulimina notovata $(3,77 \%)$ & Recurvoides scitulum $(3,57 \%)$ \\
\hline
\end{tabular}

\section{DISCUSIÓN}

La expedición CIMAR 14 Fiordos visitó las mismas estaciones que se definieron para la expedición CIMAR 2 Fiordos (1996), sin embargo no fue posible obtener muestras en todas ellas. En las 18 estaciones comunes, se observa un cambio de las especies dominantes. En CIMAR 14 las especies dominantes fueron: Uvigerina bifurcata (15,3\%), Recurvoides scitulum (10,8 \%), Melonis affinis (9,2\%), Cassidulinoides parkerianus (6,6 $\%)$, Globocassidulina minuta (5,7 \%), Oridorsalis tener (4,2 \%), Globobulimina notovata (3,8 \%), Cassidulina laevigata (3,4\%), Spiroloculina tenuis (3,3\%), Angulogerina angulosa (2,6\%), Reophax scorpiurus (2,4 \%), Involutina tenuis $(2,4 \%)$ y Rhizammina algaeformis (2\%) mientras que en CIMAR 2 dominaron: Globocassidulina rossensis (23,7\%), Angulogerina angulosa (14,8\%), Cassidulina laevigata $(7,3 \%)$, Discanomalina vermiculata $(7,1$ \%), Discorbina isabelleana (5\%), Cibicides refulgens (4,3\%), Lobatula lobatula (3,5\%), Rosalina vilardeboana (3,1\%) y Nonionella auris (2,5\%),). El aumento de foraminíferos con caparazón arenáceo y la disminución de A. angulosa y C. laevigata, dos especies típicamente marinas sugiere una condición más estuarina.

La composición faunística no reconoció nuevas especies, todos los foraminíferos recolectados en esta oportunidad, eran conocidos en canales y fiordos Hromic 2001ㄹ, 2002, 2005a², 2005b3, $2008 b^{4}, 2009^{5}$; Hromic \& Zúñiga 2003, 2005; Hromic et al. (2006). Rotaliina nuevamente es el taxa dominante tanto en número de ejemplares como de especies. Textulariina, en cambio, aumentó su abundancia en el área del $18 \%$ al 21\% de las especies. De acuerdo con Murray (1973) el dominio de estos 2 grupos es típico de ambientes de plataforma hiposalinos. Estos resultados concuerdan con los hallazgos en fiordos del hemisferio norte, en donde también es común encontrar dominando

1 Hromic, T. 2001. Foraminíferos bentónicos de canales y fiordos patagónicos chilenos. Expedición Cimar- Fiordo II. Tesis para optar al grado de Magister en Ciencias, Universidad de Concepción, Chile.

2 Hromic, T. 2005a. Asociaciones de foraminíferos bentónicos en canales y fiordos patagónicos ¿Se relaciona su distribución con las corrientes oceanográficas? Encuentro patagónico, Dirección de Relaciones Internacionales, Universidad de Magallanes. Punta Arenas, Chile.

3 Hromic, T. 2005b. Foraminíferos bentónicos: ¿Se incrementa la diversidad en función de la latitud en canales y fiordos patagónicos? Simposio Internacional New Approaches to the Quaternary Sciences in Fuego Patagonia. Centro de Estudios del Cuaternario (CEQUA) Punta Arenas, Chile.

4 Hromic, T. 2008b Foraminíferos bentónicos como indicadores biológicos de ambientes costeros, del Área Marina Costera Protegida Francisco Coloane, Magallanes, Chile. XXIII ${ }^{\circ}$ Congreso de Ciencias del Mar. Viña del Mar Chile.

5 Hromic, T. 2009 Patrones de abundancia de foraminíferos bentónicos entre 41,4ํ-43,5오 (Expedición Cimar 12 Fiordos). XXIX ${ }^{\circ}$ Congreso de Ciencias del Mar, Iquique. 
fauna arenácea en las muestras (Alve \& Nagy 1986, Korsun \& Hald 2000).

El recuento de especies manifiesta alto número de taxa con baja representación $(<2 \%)$, que no es raro en el área de estudio (Hromic 2001, Hromic et al. 2006) y que, según Murray (2006) responde a la oferta de numerosos hábitats y a la proliferación de especies oportunistas.

Si bien el análisis de Cluster manifiesta una baja similitud entre las estaciones (20\%), aún así pudo identificarse 4 grupos faunísticos que definen 4 áreas:

1) el área costera (canal Fallos, Ladrilleros) con fuerte influencia de aguas oceánicas, testimoniado por la gran abundancia de A. angulosa. Esta especie es típicamente marina y se ha observado que se mantiene en las estaciones del borde oceánico, no logrando penetrar al interior de los canales (Hromic 2007). La distribución de Oridorsalis tener, se relaciona con ambientes de aguas más profundas, y con aguas de condiciones salinas intermedias (Hromic 2001). Ishman \& Foley (1996) la recuperaron desde profundidades sobre los 1300 m en la cuenca de Canadá, en el ártico. Hromic (2009b) registra esta especie a partir de los $200 \mathrm{~m}$ de profundidad en el área al sur del Estrecho de Magallanes. Por otro lado, D. berthelothi -una especie que se adhiere al sustrato (Murray 2006)-, se asocia con aguas más turbulentas, indicaría un posible desplazamiento de masas de agua. Se le encuentra principalmente en aguas costaneras. Ha sido registrada en aguas interiores pero con menores densidades (Hromic 2001).

2) Un segundo grupo conformado por estaciones situadas en la zona sur, canales Icy, Paso del Indio, estero Falcón, presentó un número de especies y abundancia promedio más altos. Con dominio de A. angulosa y U. bifurcata, especies de hábitos infaunales, típicamente marinas, abundantes en fondos blandos lodosos- arenosos finos y que se asocian a ambientes de alta productividad o pulsos de de nutrientes (Lutze 1986). Estas especies eran conocidas en la zona costera de esta área, (Hromic et al. 2006) por lo que sugieren penetración de aguas oceánicas al interior de los fiordos. La presencia del textulárido $R$. scorpiurus, y en general de arenáceos, sugiere deterioro de las condiciones oceanográficas al interior de los canales y que podría asociarse a la influencia del Campo de Hielo Sur, entendido como una disminución de la salinidad por el aporte de aguas dulces continentales y a una alta tasa de sedimentación. Hromic (1999) y Zapata \& Moyano (1997) también registraron en las zonas internas de canales un aumento de los organismos arenáceos, que relacionaron con un intercambio escaso con aguas oceánicas.

3) El grupo № 3 conformado por el área del canal Baker y zona norte del canal Messier, tipificado por las especies $G$. notovata, $R$. contortus, y $C$. cancellata entre otras, muestra claramente la ausencia de los uvigerínidos y una disminución notable de la densidad, del número de especies y de la diversidad. En esta zona también disminuyen notoriamente las especies citadas para el Pacífico (Hromic 2011, en prensa) ${ }^{6}$. Hromic (2001) estudió los foraminíferos del canal Baker, encontrando abundantes casidulínidos: C. laevigata, C. carinata, Globocassidulina rossensis, y G. minuta, en la boca del canal, los que no lograban penetrar a su interior, y eran reemplazados por formas arenáceas. Estas especies típicamente marinas no se registraron en esta oportunidad lo que podría relacionarse con un ambiente más estuarino. Según Sievers et al. (2002) el agua estuarina (0- 50 m) saldría del Baker dividiéndose en un flujo superficial que se dirigiría hacia el golfo de Penas y uno sub-superficial que ingresaría al canal Messier. Bajo los 50 m ingresarían aguas sub-antárticas desde el golfo de Penas hacia el interior, generando un ambiente de circulación activa que probablemente sea desfavorable para el establecimiento de foraminíferos de hábitos infaunales como los uvigerínidos.

Hromic et al. (2006) demostraron que la fauna de las estaciones de la zona costera se puede diferenciar de la fauna de la zona intermedia y de los fiordos interiores. En este estudio se observó que además hay diferencias significativas, entre los canales del norte (Baker y Moraleda norte) y del sur (Concepción, Moraleda sur).

4) Un último grupo, se conforma solo por dos muestras, alejadas geográficamente por lo que este agrupamiento se presenta con reservas.

6 Hromic, T. 2011. Analisis de la comunidad foraminiferológica bentónica del seno reloncaví, islas desertores, golfo ancud y golfo corcorvado, Chile. Ciencias y Tecnología del Mar 33 (1): en prensa. 


\section{CONCLUSIONES}

a) Los foraminíferos encontrados pertenecen a la microfauna común en los canales y fiordos.

b) El suborden Rotaliina domina en cuanto a ejemplares como número de especies en el área, en cambio se observó un incremento del número de ejemplares de Textulariina en relación con los resultados obtenidos por la expedición CIMAR 2 Fiordos.

c) Las especies con mayor abundancia fueron U. bifurcata, Globobulimina notovata que también mostraron gran distribución en el área. Se identificaron 4 áreas faunísticas: la primer área representada por las siguientes especies indicadoras: C. variabilis y $N$. auris. La segunda, por O. tener, $S$. tenuis, $A$. angulosa y $D$. bertheloti. La tercera, por A. angulosa, U. bifurcata, $R$. scorpiurus, $D$. consobrina y G. minuta. Y finalmente la cuarta, por $G$. notovata, $R$. contortus, $C$. cancellata, Nonionella sp. a y $M$. affinis.

\section{AGRADECIMIENTOS}

La autora agradece al Comité Oceanográfico Nacional (CONA) la oportunidad de obtener muestras de la expedición CIMAR 14 Fiordos. A la Universidad de Magallanes, el apoyo para realizar este trabajo. A la Srta. Lyta Quezada, su dedicación e interés en los foraminíferos. Al Dr. Américo Montiel, su aporte en los cálculos estadísticos y, en general, a todos aquellos que contribuyeron a que este trabajo se terminara.

\section{LITERATURA CITADA}

Alve, E. \& J. Nagy 1986. Estuarine foraminiferal distribution in Sandebukta, a branch of the Oslo Fjord. Journal of Foraminiferal Research 16 (4):261-284

Boltovskoy, E. 1965. Foraminíferos recientes. Eudeba. Buenos Aires. 510 pp.

Bray, J.R. \& J.T. Curtis 1957. An ordination of the upland forest communities of Southern Wisconsin. Ecological Monographs 27:325349

Clarke, K.R. 1993. Non parametric multivatiate analyses of changes in communitiy structure. Austral Journal Ecology 18:117-143
Clarke, K. \& R. Gorley 2006. Primer v. 6 User Manual/Tutorial PRIMER-E Ltd.: Plymouth Marine Laboratory UK 190 pp.

Clarke, K.R. \& R.H. Green 1988. Statistical design and analysis for a biological effects study. Marine Ecology Progress Series 46:213-226

Clarke, K.R. \& R.M. Warwick 1994. Change in marine communities: an aproach to statistical analysis and interpretation. Plymouth Marine Laboratory.

Kruskal, J.B. \& F. Carmone 1971. How to use MDSCAL (Version 5M) and other useful information. Bell Telephone Laboratories, 600 Mountain Ave., Murray Hill, New Jersey, USA 07974

Ellis, B. \& A. Messina 1940. Catalogue of Foraminifera. American Museum of Natural History. Sp Pub. 30

Loeblich, A. \& H. Tappan 1988. Foraminiferal Genera and Their Classifications. Van Nostrand Reinhold Co. N.Y. Text- vol: 970. Pl-Vol. 212 pp. 847

Ludwig, J.A. \& J.F. Reynold 1988. Statistical Ecology. J. Wiley \& Sons. New York 337 pp.

Hromic, T. 1999. Foraminíferos bentónicos de canales australes de Kirke: canal Kirke, golfo Almte. Montt y seno Última Esperanza, XII ${ }^{a}$ Región, Magallanes y Antártica chilena. Anales Instituto Patagonia Ser. Cs. Nat. (Chile) 27:91-104

Hromic, T. 2001. Foraminíferos bentónicos del canal Baker (47옥 74ํW) Pacífico sudoriental, Chile. Anales Instituto Patagonia Serie. Cs. Nat. (Chile) 29:135-156

Hromic, T. 2002. Foraminíferos bentónicos de bahía Nassau, Cabo de Hornos, Chile. Comparación con foraminíferos del cono sur de América, Antártica y Malvinas. Anales del Instituto de la Patagonia Serie. Cs. Nat. (Chile) 30:95-108 Hromic, T. 2005. Foraminíferos bentónicos entre seno Reloncaví y Golfo Corcovado. Crucero CIMAR 10 Fiordos, Informes preliminares Comité Oceanográfico Nacional-Chile pp. $125-134$

Hromic, T. \& M. Zúñiga 2003.- Diversidad, abundancia y distribución de la superfamilia Buliminacea Jones, 1875 , en canales y fiordos patagónicos, Pacífico Sudoriental. Anales Instituto Patagonia (Chile) 31:55-74 
Hromic, T. \& M. Zúñiga 2005. Foraminíferos bentónicos de ambientes someros extraídos durante la Expedición CIMAR 7 Fiordos, canales patagónicos (42-47은 $\mathrm{S})$. Boletín de la Sociedad de Biología de Concepción, Chile 76:25-38

Hromic, T., S. Ishman \& N. Silva 2006. Benthic foraminiferal distributions in Chilean fjords: $47^{\circ} \mathrm{S}$ to $54^{\circ} \mathrm{S}$ Marine Micropaleontology 115-134.

Hromic, T. 2007. Biodiversidad y ecología del microbentos (Foraminifera: Protozoa) entre la boca del Guafo y golfo de Penas (43-46S), Chile. Ciencia y Tecnología del Mar 30(1):89-103.

Hromic, T. 2009. Estructura comunitaria del taxa Foraminiferida (Protozoa) según profundidad, en el fiordo Comau, Chiloé, Chile. Anales Instituto Patagonia (Chile) 37(1):39-51.

Hromic, T. 2009b. Distribución batimétrica de foraminíferos bentónicos (Protozoa: Foraminiferida) al sur del estrecho de Magallanes (52-56으), Chile. Anales Instituto Patagonia (Chile) 37(1):23-38.

Ishman, S. \& K. Foley 1996. Modern Benthic Foraminifer Distribution in the Amerasian Basin, Arctic Ocean. Micropaleontology 42(2):206-220

Korsun, S. \& M. Hald 2000. Seasonal dynamics of benthic foraminifera in a glacially fed fjord of Svalbard, European Arctic. Journal of Foraminiferal Research 30(4):251-271

Lutze, G.F. 1986. Uvigerina species of the Eastern North Atlantic. Utrecht Micropaleontological Bulletins 22(35):21-46

Marchant, M. 1993 Foraminíferos de la Bahía Scholl, Región Magallánica, Chile (Protozoa: Foraminifera) Gayana Zoología 57(1):61-75

Magurran, A.E. 1988. Ecological diversity and its mesasurement. Princeton University Press. New Jersey 179 pp.
Moreno, C.E. 2001. Métodos para medir biodiversidad. M \& T-Manuales y Tesis SEA, Vol. 1. Zaragoza. $84 \mathrm{pp}$.

Murray, J. 1973. Distribution and ecology of living benthic foraminiferas. Heineman (ed.) London. $274 \mathrm{pp}$.

Murray, J. 2006 Ecology and applications of Benthic foraminifera Cambridge Univ. Press.

Sievers, H., C. Calvete \& N. Silva 2002. Distribución de características físicas, masas de agua y circulación general para algunos canales australes entre el golfo de Penas y el estrecho de Magallanes (Crucero CIMAR -Fiordo 2) Chile. Ciencia y Tecnología del Mar 25(2):17-43.

Silva, N., C. Calvete \& H. Sievers 1998. Masas de agua y circulación general para algunos canals australes entre Puerto Montt y Laguna San Rafael, Chile (Crucero CIMAR 1 Fiordos). Ciencia y Tecnología del Mar 21:17-48.

Violanti, D., B. Loi \& R. Melis 2000. Distribution of Recent Foraminifera from the Strait of Magellan. First quantitative data. Bollettino della Museo Region Scieta della Natural., Torino Italy 17(2):511-539.

Zapata, J. \& H. Moyano 1997 Foraminíferos bentónicos recientes de Chile Austral. Boletín de la Sociedad Biología, Concepción, Chile 68:27-37.

Zapata, J., C. Zapata \& A. Gutiérrez 1995. Foraminíferos bentónicos del sur de Chile. Gayana Zoología 59 (1):23-40.

Wood-Walker, R.P. Ward \& A. Clarke 2002. Largescale patterns in diversity and community structure of surface water copepods from the Atlantic Ocean. Marine Ecology Progress Series 236:189-203. 
